\title{
On the relationship between black hole mass and X-ray variability amplitude in the low-mass regime of active galactic nuclei
}

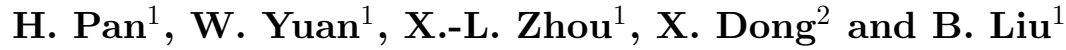 \\ ${ }^{1}$ National Astronomical Observatories, Chinese Academy of Sciences, 20A Datun Road, \\ Chaoyang District, Beijing, China \\ email: panhaiwu@bao.ac.cn \\ ${ }^{2}$ Yunnan Observatories, Chinese Academy of Sciences, Kunming, Yunnan, China
}

\begin{abstract}
Recent studies of active galactic nuclei (AGN) found a statistical inverse scaling between the X-ray normalized excess variance $\sigma_{\mathrm{rms}}^{2}$ (variability amplitude) and the black hole mass spanning over $M_{\mathrm{BH}}=10^{6}-10^{9} M_{\odot}$. We present a study of this relation by including AGN with $M_{\mathrm{BH}}=10^{5}-10^{6} M_{\odot}$. It is found that the relation is no longer a simple extrapolation of the known inverse proportion, but starts to flatten around $10^{6} M_{\odot}$. This behavior can be understood by the shape of the power spectrum density of AGN and its dependence on the black hole mass.
\end{abstract}

Keywords. galaxies: active, galaxies: nuclei, X-rays: galaxies

\section{Introduction}

Recent studies suggested an inverse linear correlation between black hole $(\mathrm{BH})$ mass and excess variance $\left(\sigma_{\mathrm{rms}}^{2}\right)$ in logarithmic space for active galactic nuclei (AGN) with $M_{\mathrm{BH}}=10^{6}-10^{9} M_{\odot}$ (Zhou et al. 2010; Ponti et al. 2012). This scaling relation is tight enough to provide a novel method to estimate the black hole mass of AGN. However, it is not clear if this relation can be extended to lower black hole masses. Our work is to study the relation in the low-mass regime using a sample of low-mass AGN with $M_{\mathrm{BH}}<2 \times 10^{6} M_{\odot}$ observed with XMM-Newton and ROSAT.

\section{Result and discussion}

We compiled a sample of 15 low-mass AGN from our sample of low-mass AGN with $M_{\mathrm{BH}}<2 \times 10^{6} M_{\odot}$ (Dong et al. 2012). There are 11 objects observed with XMM-Newton for a total of 15 observations, and 5 objects observed with ROSAT for 6 observations (one object observed with both satellites). The accretion rates in the Eddington units span the range $0.06-0.90$. We calculate the X-ray excess variances (Nandra et al. 1997) and normalize their values on a timescale of $80 \mathrm{ks}$, using the three power spectrum density (PSD) models in Table 1 (see Papadakis 2004 for the details about the PSD shape of AGN). The relation of the excess variance and black hole mass is plotted in Figure 1 . It shows that for almost all the sources with $M_{\mathrm{BH}}<2 \times 10^{6} M_{\odot}$, the excess variances fall systematically below the extrapolation of the $M_{\mathrm{BH}}-\sigma_{\mathrm{rms}}^{2}$ relation derived from the highmass sample (Ponti et al. 2012). In fact, the $M_{\mathrm{BH}}-\sigma_{\mathrm{rms}}^{2}$ relation can be derived from the dependence of the break frequency of the PSD, since excess variance is the integral of the PSD over frequency domain (van der Klis 1989, 1997). We fix the observed $\nu_{\mathrm{br}}\left(M_{\mathrm{BH}}, \dot{m}\right)$ relations as their original form (McHardy et al. 2006; González-Martín \& Vaughan 2012), 


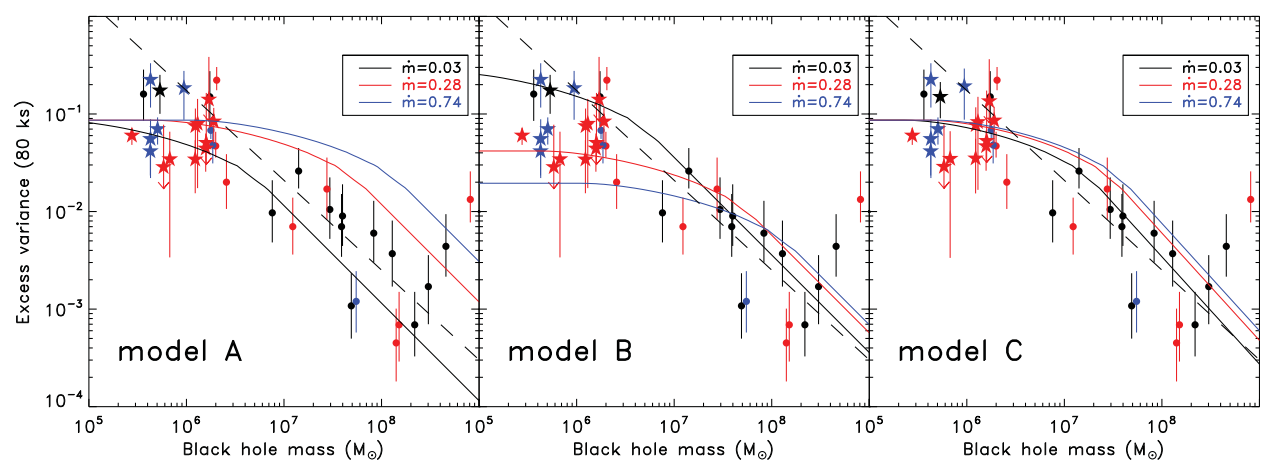

Figure 1. Relationship of the black hole mass and the excess variance for the objects in our sample (stars), together with the sample (dots) and the best-fit relations (dashed lines) from Ponti et al. (2012). The objects with $\dot{m}$ in the three $\dot{m}$ bins $(0.01-0.1,0.1-0.5$, and $0.5-1.0)$ are plotted in black, red and blue, respectively. The solid lines represent the relations derived from the best-fit PSD models for three accretion rates.

Table 1. PSD models used

\begin{tabular}{|c|c|c|c|c|}
\hline Model & Break Frequency Formalism & $\left|\begin{array}{c}\text { PSD Amplitude } \\
\text { Suggested in Previous Work }\end{array}\right|$ & $\left|\begin{array}{c}\text { PSD Amplitude } \\
\text { Fitted in this Work }\end{array}\right|$ & $\chi^{2} / d o f$ \\
\hline$|\operatorname{model} \mathrm{A}|$ & $\begin{aligned} \nu_{\mathrm{br}}= & 0.003 \dot{m}\left(M_{\mathrm{BH}} / 10^{6} M_{\odot}\right)^{-1} \\
& \text { McHardy et al. }(2006)\end{aligned}$ & $\begin{array}{c}0.017 \pm 0.006 \\
\text { Papadakis }(2004)\end{array}$ & $C_{1}=0.017 \pm 0.002$ & $150 / 36$ \\
\hline model B & $\begin{aligned} \nu_{\mathrm{br}}= & 0.003 \dot{m}\left(M_{\mathrm{BH}} / 10^{6} M_{\odot}\right)^{-1} \\
& \text { McHardy et al. }(2006)\end{aligned}$ & $\left|\begin{array}{c}\alpha=0.003_{-0.001}^{+0.002} \\
\beta=0.8 \pm 0.15 \\
C_{1}=\alpha \dot{m}^{-\beta} \text {, Ponti et al. }(2012)\end{array}\right|$ & $\begin{array}{c}\alpha=0.010 \pm 0.001 \\
\beta=0.40 \pm 0.04\end{array}$ & $106 / 35$ \\
\hline $\mid \operatorname{model~C|}$ & $\begin{array}{l}\nu_{\mathrm{br}}=0.001 \dot{m}^{0.24}\left(M_{\mathrm{BH}} / 10^{6} M_{\odot}\right)^{-1} \\
\text { González-Martín \& Vaughan }(2012)\end{array}$ & $\begin{array}{c}0.017 \pm 0.006 \\
\text { Papadakis }(2004)\end{array}$ & $C_{1}=0.015 \pm 0.002$ & $102 / 36$ \\
\hline
\end{tabular}

and obtain the PSD amplitude by fitting the $M_{\mathrm{BH}}-\sigma_{\mathrm{rms}}^{2}$ relation. It can be seen that all the three models can reproduce the observed trend of the $M_{\mathrm{BH}}-\sigma_{\mathrm{rms}}^{2}$ relation well (Figure 1). In conclusion, the inversely proportional $M_{\mathrm{BH}}-\sigma_{\mathrm{rms}}^{2}$ relation established in the high-mass regime fails to extend to $M_{\mathrm{BH}}$ below $10^{6} M_{\odot}$. Our result is in good agreement with that obtained from a recent similar study by Ludlam et al. (2015). This is also consistent with the model prediction from our current understanding of the PSD of AGN and the dependence of the break frequency on $M_{\mathrm{BH}}$.

\section{Acknowledgements}

This work is supported by grants No. XDB09000000 \& No. 11473035

\section{References}

Dong, X.-B., Ho, L. C., \& Yuan, W., et al. 2012, ApJ, 755, 167

González-Martín, O. \& Vaughan, S. 2012, A\&A, 544, A80

Ludlam, R. M., Cackett, E. M., \& Gultekin, K., et al. 2015, MNRAS, 447, 2112

McHardy, I. M., Koerding, E., Knigge, C., Uttley, P., \& Fender, R. P. 2006, Nature, 444, 730

Nandra, K., George, I. M., Mushotzky, R. F., Turner, T. J., \& Yaqoob, T. 1997, ApJ, 476, 70

Papadakis, I. E. 2004, MNRAS, 348, 207

Ponti, G., Papadakis, I., \& Bianchi, S., et al. 2012, A\&A, 542, A83

van der Klis, M. 1989, Timing Neutron Stars, 27

van der Klis, M. 1997, Statistical Challenges in Modern Astronomy II, 321

Zhou, X.-L., Zhang, S.-N., Wang, D.-X., \& Zhu, L. 2010, ApJ, 710, 16 